Int Arch Allergy Immunol 1997;114:407-408

\title{
Subject Index, Vol. 114, 1997
}

International Archives of

Allergy $^{\wedge}$ Immunology

Supplement 1 has its own Subject Index

Adducts 46

Adhesion molecules 46, 86 Agglutination, tissue particles 343 Airway responsiveness 285

Allergic contact dermatitis 298 Allergy 591

- $\quad$ type I 272

Allosensitization 303

Allotype 116

Amplification refractory mutation system

- polymerase chain reaction 94 Antibodies 103,246 Antigen provocation 81 Aspergillus 205

Asthma attack, spontaneous 373 -, elderly patients 379 Atherosclerosis 10, 246 Atopy 193

Autoantibodies 323 Autoimmunity 246 Azelastine 185,285

Baculovirus 229 Baker's asthma 278 Basophil(s) 68, 207, 348 Bermuda grass 265

- pollen 258 Betvl 175,272

- 2272 Blood products 303

transfusion 303 Bronchial asthma 97, 285, 373

biopsy 285 Budesonide 185

$\mathrm{Ca} 2+$ binding allergen 265 Calcium ion Ill Carbohydrate-deficient glycoprotein

syndrome 116 Carbohydrates 116 Cell adhesion 167

- $\quad$ injury Ill, 336

Cell-mediated immunity 90

Chagas' disease 103

Characterization 251

Charcot-Leyden crystal protein 130

Choroid 139

Chronic granulomatous disease 144 Chronic-relapsing experimental allergic

encephalomyelitis 74 Chymase 207 Cloning 229

Cold stimulation test 400

- urticaria 400

Covered and heated swimming-pool 205 Cross-reactivity 251 Cyclic AMP 348

- $\quad$ AMP-dependent protein kinase Ill,

336

Cynomolgus monkey 229 Cysteinyl leukotriene 97

- $\quad$ - receptor antagonist 97

Cytokine(s) 10, 30, 90, 103, 153, 237, 323

Cytotoxic T lymphocytes 120

11-Dehydrothromboxane B2 373 
Dendritic cell 30

Derf2 354

Dermatophagoidespteronyssinus 59

Dexamethasone 74

Diagnosis in vitro 361

Diesel exhaust particulates 167, 237

Drug hypersensitivity 361

Eicosanoids 153 Elastin peptides 218 Elastin-laminin receptor 218 Electron microscopy 1

Endothelial cell 167

-, human 336 Enzyme-amplified ELISA 175 Eosinophil(s) 167, 185

cationic protein 130

progenitors 130 Epidemiology 193 Epithelial cell 167 Epitope specificity 246 E-selectin 86

Exposure, airborne 278 Extract 251

Fatty-acid composition 153 FceRI 161

Flow cytometry 38, 144 Follow-up 193 Formaldehyde 237 Fungi 205

Gingivitis 246 Glucocorticoids 74 Glycoconjugates 103 Glycoprotein 116 Granules 130 Group I allergen 258

Hair dye 298 Halothane 46 Hay fever 193 Heat shock protein 246 Helper T cell 30 Henna 298

Hepatitis 46 Histamine 1, 207

- $\quad$ release 68

HIV-1 120

HLA 354

HLA-DR 15

Honey 385

House dust mite 59, 202 Human 139

endothelial cells Ill

neutrophils Ill Humoral antibody response 385 Hyperresponsiveness 389

-, bronchial 379 Hypersensitivity 46

- $\quad$ pneumonitis 205

ICAM-1 86 IgD 54 IgE 59

antibodies 367 IgG antibodies 367

subclasses 175 Immediate hypersensitivity 193 Immune activation 54

deviation 30

regulation 303 Immunity 175 Immunoblotting 367 Immunodeficiency 54 Immunodiffusion 385

Immunodominant peptides 272 Immunoelectron microscopy 130 Immunoglobulin 116

preparations, human intravenous 323 Immunohistochemistry 285 Immunopathology and reactivation of

infection 103 Immunophenotype 38 Immunosuppression 385 Immunotherapy 23 Infants 175

Infection 116 Inflammation 10,389 Inhalation 389 Interferon- $\gamma$ 120,229

KAÏIGER,

E-Mail karger@,karger.ch Fax+4161306 1234 http://www. karger. ch

C1997S. KargerAG, Basel

407

Interleukin-2 316,323

Interleukin-4 237, 348 
Interleukin-4,-5,-6 81

Interleukin-6 237

Interleukin-7 120

Interleukin-13 348

Intracellular adhesion molecule-1 329

Jurkat cells 316

KF19514 389 Kupffer cells 46 Kynurenine 224

LDL 10

Leukocyte filtration 303 Leukotriene C4 207

- $\quad$ E4 373

Lymphocytes, human 218 -, tumor-infiltrating 38 Lysozyme 202

Macrolide Ill

Magnetic beads 316

Major basic protein 130

Mastcell(s) 1, 10, 139, 161,207,237

Mediator release 139

Mediators 185

Mifepristone 74

Monoclonal antibody 258

Monocyte, human 336

Mosquito allergy 367

Mouse 74

MSP-1 15

Muramyl dipeptide 23

Nasal allergen challenge 185

allergy 167

hypersensitivity 167

mucosa 81 Natural Derp 2202 Neurospora 205 Neutrophil 144

-, human 336 Nickel sensitivity 94 Nitric oxide Ill, 336 Non-small-cell lung cancer 38

Nonsteroidal anti-inflammatory drugs 361

Oilseed rape 265 Organ specificity 343

Passive cutaneous anaphylaxis 161,385 Peak expiratory flow 97 Pentoxifylline 329 Peptide 354

Perennial allergy 81 Phosphodiesterase 348, 389

3 inhibitor 379 Plasma histamine 400 Plasmodium falciparum 15 Platelet-activating factor 389

Pollen 251

allergen 265

Polymorphonuclear granulocytes 329 Postembedding gold labeling methods 1 Pranlukast 97

Prognostic factors 38 Protection 293 P-selectin 86 Pulmonary function 285 Putranjiva roxburghii 251

Rat basophilic leukemia cell 161 Recombinant allergens 59, 68

- $\quad$ Derp2 202

Reliability 193

Respiratory oxidative burst 144 Reverse passive cutaneous anaphylaxis 161 Rhinitis 193

Ricinus communis 251 RU486 74

Saccharomyces cerevisiae 202 Saliva 367 Saponin 153 
Schizosaccharomyces pombe 15 Seasonal allergic rhinitis 185 Secretagogues 139 Secretion 1 Sepsis 329

Sequential epitope 258 Serum 116 Sesame seed oil 153 Signal transduction 316 Soluble CD23

120 Species specificity 343

Staphylococcus 329 Streptococcal erythrogenic toxin 224 Stress protein 246 Sulfidoleukotrienes

361 Superantigen 224 Superoxide Ill

- $\quad$ anion 23

Suspensions of pulverized organs 343 Symptom score 97, 285

Tcell(s) 120

- antigenicity 272

- clone 15

- epitope 354

epitope 15 Tachykinin 389 TAP1 94 TAP2 94

Thermophilic actinomycetes 205

Thy-1 161

Tobacco smoking 193

Total IgE 193

Transfusion side effects 303

Trypanosoma cruzi 103

Tryptase 207

Tryptophan 224

Tuberculosis 23

Tumor necrosis factor 329

- $\quad$ - factor- $\alpha 323$

Upper airway disease 193 Urticaria 86

Vaccine 90, 293 VCAM-1 86

Verocytotoxin·VTl·VT2 293 Viral transmission 303 VT-liposome 293

Wheat allergen 278

- $\quad$ flour $\alpha$-amylase inhibitor 278

X-linked agammaglobulinemia 90 Yeast, baker 202

408

Int Arch Allergy Immunol Vol. 114, 1997

Subject Index 